\title{
Pratiques religieuses africaines et médias numériques
}

\author{
Prolonger la réflexion
}

\begin{abstract}
Katrien Pype ${ }^{1}$
Cette série de six articles, tous focalisés sur l'Afrique francophone, traite chacun à leur manière du thème principal de l'usage de l'Internet par des groupes religieux sur le continent africain. Cinq de ces contributions explorent l'usage religieux des médias numériques en Afrique de l'Ouest alors que la dernière se concentre sur l'Afrique centrale. Nous en tirons une vue d'ensemble équilibrée sur diverses communautés religieuses telles le Hibut-Tarqiyyah du Sénégal, des églises chrétiennes de Ouagadougou, des chrétiens de la République démocratique du Congo, des fidèles camerounais du pasteur nigérian T. B. Joshua ou encore des musulmans ivoiriens hors ligne comme en ligne ainsi qu'une communauté mixte regroupant des chrétiens et des musulmans au Burkina Faso. Les thématiques dominantes abordées sont: la représentation en ligne; le numérique en tant qu'espace étendu d'apprentissage et de conversion religieuse ; la religion et la sphère publique ; et, pour terminer, les formes de religiosité en Afrique subsaharienne. Cette compilation d'articles constitue un complément utile à l'abondante littérature anglophone sur les mondes médiatiques africains et leurs enchevêtrements religieux (voir entre autres de Witte, 2011; Hackett et Soares, 2014; Graetz, 2014 ; Meyer, 2015 ; Pype, 2012 ; Schulz, 2011).

Parmi les analyses fournies, voici les trois thèmes qui m'ont frappée par leur pertinence et leur forte connexion avec les recherches actuelles sur les communautés religieuses en ligne sur le continent africain et au-delà : (a) l'importance de « réseaux » religieux; (b) l'interaction entre le religieux et la politique; (c) les pratiques de foi en ligne et leur dialectique avec la politique de la religion (particulièrement en termes d'autorité, de distribution et de transmission de la connaissance religieuse).
\end{abstract}

\section{« Réseaux » religieux}

La plupart des auteurs mettent en exergue les interactions entre individus géographiquement éloignés. Dès lors, nous nous trouvons confrontés aux « opportunités » offertes par les modalités de déterritorialisation qu'offrent les réseaux sociaux. Ces op-

\footnotetext{
${ }^{1} \mathrm{KU}$ Leuven et Université de Birmingham.
} 
portunités posent des défis aux chercheurs. Par exemple, comment définir les communautés religieuses étudiées si un site web chrétien du Burkina Faso présente le travail de pasteurs internationaux (Gosselin) ? Ou encore, comme l'évoque Makal, comment interpréter l'appartenance d'un internaute lorsque nous lisons les commentaires de Congolais (ou d'individus qui s'identifient comme Congolais bien qu'ayant acquis la nationalité française, britannique, sud-africaine ou autre) sur la page web d'un magazine politique français, mobilisant des discours religieux pour évaluer la gouvernance congolaise ou des événements politiques? Les diasporas et les connexions transnationales apparaissent dans chacun des articles présentés. Évidemment, la participation à un réseau numérique transnational ne se limite nullement aux sphères religieuses. Elle soulève néanmoins des interrogations quant à l'expérience de la religion, l'interaction entre coreligionnaires et la signification de la distance versus la proximité dans notre monde globalisé. Bien que les réseaux sociaux soient toujours localisés (en ce sens que les individus sont en réalité présents dans un certain espace physique lorsqu'ils interagissent avec les contenus en ligne et les périphériques qui permettent ces interactions), les distances sont comblées, affectant la circulation des opinions, attentes, propos, images ou sons et leur négociation dans des espaces distants.

Concernant l'engagement des diasporas dans des conversations numériques, il est intéressant de s'inspirer des réflexions sur le nationalisme et le numérique en Afrique. En particulier, dans sa tranchante analyse de la sphère politique publique érythréenne, qui intègre la communication et le partage d'informations en ligne comme un élément crucial, Bernal (2014) décrit la façon dont l'État-nation érythréen est l'objet de nombreuses discussions et prend corps dans les mondes vécus de la diaspora. L'influence d'Internet, de la diaspora et de la mobilité toujours croissante des individus conduit la chercheuse à une critique de la fameuse notion andersonienne de communauté (Anderson, 1983), qui suggère des frontières définies, une stabilité et un centre. Selon Bernal, le terme « réseau » s'avère plus approprié, car il permet d'inclure l'interactivité de citoyens ordinaires et la contribution de personnes résidant en dehors du territoire national dans la formation de l'idée de la nation érythréenne. Dans la présente collection d'articles, les auteurs présentent des fragments sur les modalités de réception dans des contextes diasporiques et "domestiques » et sur la manière dont des personnes interagissent avec leurs condisciples musulmans distants. Il me semble que la notion du "réseau religieux » permettra d'inclure cette interactivité dans l'analyse des formations religieuses et l'expérience sociale du religieux dans cette ère numérique.

En plus, il existe un besoin urgent de retracer les connexions de réseau au-delà du nœud « Afrique-Nord Globalisé ». Au cours de mes propres recherches sur la production médiatique d'un prophète catholique marginal de Kinshasa (Pype, 2018), j’ai pu observer comment des connexions entre apparitions mariales à Kibero (Rwanda), Fatima (Portugal) et Lourdes (France) sont visualisées et mises en œuvre (par le biais de 
liens hypertextes) sur le site web du prophète. L'espace numérique devient dès lors une plateforme sur laquelle le prophète s'inscrit dans un monde d'apparitions mariales reconnues par le monde catholique, alors que ses propres récits d'apparitions de la Vierge sont dénigrés à Kinshasa, considérés comme faux, ou pire, perçus comme les affirmations d'un fou. Il me semble que la comparaison des travaux numériques musulmans du continent africain avec ceux de publics islamiques d'Indonésie, et plus largement d'Asie du Sud, ouvre un nouveau champ d'analyse qui se situe au nœud de la mobilité religieuse et des cultures de la technologie.

Une approche des communautés religieuses comme "réseaux » chrétiens ou musulmans ne nie cependant pas la pertinence des activités religieuses hors ligne. Audelà d'une étude des «e-esthétiques» de la communication religieuse numérique, il convient également d'observer la façon dont les personnes usent des médias numériques. Dans une fascinante étude, Batibonak et Batibonak nous montrent comment des groupes se forment dans et par la réception de médias numériques. En établissant un lien étroit avec les "clubs d'écoute", réunions autour d'un poste de radio pour écouter des émissions et discuter de la pertinence du contenu des émissions, Batibonak et Batibonak évoquent des rassemblements à Yaoundé dans la maison d'une femme qui peut se permettre une connexion Internet. De petits groupes de chrétiens néo-pentecôtistes camerounais se recueillent ainsi dans des espaces domestiques, pour prier, chanter, pratiquer des cultes et visionner des émissions du pasteur nigérian T. B. Joshua sur sa chaîne de télévision numérique (disponible sur YouTube), participant à distance aux cultes de guérison organisés par cette Église nigériane. De façon similaire, des webinaires organisés via Skype ne sont pas consommés individuellement, mais collectivement. En contraste, les communications par WhatsApp sur des sujets religieux peuvent être tenues en solitaires, sans la présence physique d'autres. Sur ces plateformes de réseaux sociaux, le groupe est virtuellement présent. Le réseau est toujours au-delà.

Pourtant, si l'idée d'un « réseau » sous-tend une absence de hiérarchie et évoque une structure «rhizomateuse », l'analyse de Binaté de groupes Facebook islamiques ivoiriens introduit une forme résolument hiérarchique de communauté dans la discussion sur la religion et les médias. En particulier, le groupe Facebook «Fan Club Prophète Muhammad» suggère que l'activité médiatique religieuse s'inspire davantage de la culture de la célébrité que de publics nationaux. En plus, des leaders pluriels peuvent littéralement diriger la communauté : la célébrité (de manière symbolique), les leaders religieux et/ou des administrateurs réseaux avec une affiliation religieuse. De manière frappante, dans le cas présent, les fidèles sont non seulement des membres d'une religion, mais ils sont aussi littéralement des «followers» en ligne, un concept utilisé pour indiquer une connexion en ligne; ils se définissent également eux-mêmes comme des "fans ». L'interaction sociale au sein de cette communauté religieuse est empreinte d'un langage hybride de mondes religieux, numériques et de 
célébrité. De nouvelles formes de vénération, d'identité religieuse et de leadership en émergent.

\section{Groupes religieux et politiques nationales}

Malgré le fait que des spécialistes des médias et de la communication (entre autres Appadurai, 1996) reconnaissent qu'une des principales forces des nouveaux médias est leur caractère déterritorialisé, nous observons que tous les auteurs se concentrent sur les communautés nationales. S'ils reconnaissent tous le rôle des diasporas dans les publications en ligne, dans les communications et parfois même dans l'établissement de pages web et des groupes de discussion, les auteurs cantonnent la pertinence de leurs conclusions à un niveau national.

Dès lors, ces contributions soulèvent une importante tension entre deux échelles alors qu'il semble que l'État-nation semble avoir gagné en pertinence dans l'établissement de communautés virtuelles de médias religieux. L'État-nation fait-il un grand retour? Ou, peut-être il n'a jamais vraiment disparu. En s'exposant à la sphère publique et en engageant les consommateurs en ligne, les groupes religieux se déplacent de plus en plus dans le domaine politique et s'engagent dans des questions publiques et nationales. À cet égard, il est fascinant de se pencher sur le rôle qu'ont acquis les leaders religieux dans la résolution de conflits au Burkina Faso, ainsi que le montre Kaboré. Sous l'impulsion des autorités de l'État, les leaders catholiques et musulmans font alliance pour lutter contre Boko Haram et le terrorisme. Pour ce faire, des publications en ligne documentent la solidarité entre communautés religieuses dans l'espoir d'influencer les internautes et de réduire les antagonismes entre chrétiens et musulmans.

La question de l'échelle se pose également à un autre niveau : la plupart des articles ont trait aux minorités, qu'elles soient religieuses ou politiques (c'est-à-dire l'opposition). Ceci pose la question du pouvoir et d'Internet. Pourquoi ces minorités de toutes sortes sont-elles si avides de communiquer sur Internet? Les données de recherche présentées dans ces six articles nous suggèrent que le cyberespace est devenu un espace d'émancipation pour les pauvres, les sans-voix, les opprimés et les marginalisés. Si les élites religieuses et politiques investissent davantage dans les médias classiques tels la radio et la télévision, elles pourraient avoir négligé le potentiel social et politique du numérique. Makal écrit que l'opposition a été active en ligne bien avant l'État congolais. De nombreuses observations similaires ont été faites au sujet de minorités religieuses où l'Église catholique domine la sphère publique. De toute évidence, ces observations doivent être appuyées par des preuves plus empiriques sur des environnements médiatiques locaux. Elles sont néanmoins déjà significatives puisqu'elles mettent à mal les idées préconçues sur le pouvoir et l'accès aux médias. Il importe de se demander qui (et de quelle manière) a ouvert l'espace numérique aux démunis, et comment l'activité, la contestation et la provocation numériques dans des 
sphères à la fois religieuses et politiques présentent des impacts sur la répartition du pouvoir, les jeux d'influences dans les espaces décisionnels, la médiation et la sanction de conflits. D'ailleurs, tous les articles soulignent également le rôle de la censure ; il est donc évident qu'Internet n'est pas un espace de liberté absolue. En effet, nous nous trouvons dans une situation où les États-nations africains ont eu le temps de s'ajuster aux développements technologiques et ont pu mettre en place leurs propres schèmes de surveillance, d'accès et de contrôle.

\section{Participation web et connaissance sacrée}

Le troisième grand fil conducteur de ces articles concerne la répartition numérique des connaissances religieuses. Les sites web islamiques et (néo)-pentecôtistes deviennent ce que Sylla appelle «des lieux de culte virtuels». Sylla considère le site web mouride sénégalais htcom.sn comme une école coranique virtuelle (daara virtuelle), où commentaires coraniques, chants et prières sont enseignés. De même, un pasteur burkinabè est actif sur la plateforme Topchrétien, qui rassemble les Églises évangéliques du monde francophone, proposant quotidiennement des versets bibliques sur leur mur Facebook et leur compte Twitter (Gosselin). Cette offre publique de connaissances religieuses soulève des questions intéressantes sur les frontières entre différentes sphères de connaissance. Dans la mesure où les experts sont en possession de savoirs parfois jugés proscrits aux non-initiés, je demande à quelle(s) échelle(s) les leaders, influences et disciples religieux exploitent les nouveaux médias pour agir à différents niveaux de la société. L’un des principaux éléments mis en évidence par le groupe de recherche sur les réseaux sociaux (Miller et al., 2016) dans neuf lieux différents dans le monde est que les différentes plateformes de réseaux sociaux sont utilisées de façons différentes, pour interagir dans des modalités differentiées avec « des Autres (religieux/sociaux) ». Par exemple, les enfants dans un village anglais utiliseront Instagram pour se connecter à leurs camarades de classe, tandis qu'ils publieront des contenus accessibles à leurs parents, gardiens ou à des étrangers sur Facebook.

J'entrevois trois raisons pour dresser une échelle dans les mondes médiatiques numériques religieux : (a) la connaissance religieuse n'est jamais totalement transmise au groupe de fidèles ; la connaissance sacrée se distribue plutôt de façon inégale aux différents niveaux de la hiérarchie religieuse, sur différentes plateformes, avec des modalités respectives d'accès et de distribution pouvant perpétuer des sphères de confidentialité et d'accès inégal au savoir. Ceci nous amène à des réflexions fascinantes comme: quels sont les médias numériques que les imams utilisent entre eux pour informer, débattre et commenter? Comment maintiennent-ils des barrières ? Comment opèrent-ils la transition d'une échelle à une autre ? Comment cette distribution des connaissances est-elle reflétée et maintenue dans les communications par les médias numériques? Ou, comment s'échelonne le conflit religieux dans le monde numérique ? En effet, les rivalités et concurrences religieuses se déroulent aussi dans 
les sphères audiovisuelles et numériques. Les attaquants font face à deux défis : ils doivent prouver qu'ils sont de bons leaders, dignes de confiance, surpassant les leaders qu'ils attaquent. En plus de prouver cela, ils doivent dénigrer, exposer et dénoncer leurs adversaires. Il est prévisible que cela survienne sur diverses plateformes puisque des publics différents y sont ciblés. En plus, dans la mesure où les adeptes s'expriment sur des questions de politique nationale, comment contournent-ils la surveillance politique? Comment se prémunissent-ils de répercussions des services secrets ou d'autres agences d'État qui punissent et disciplinent les voix de l'antiestablishment?

Certains des articles réunis ici abordent aussi (b) la question du ludique et du divertissement. En effet, des contenus de divertissement sont également partagés sur les plateformes numériques religieuses, tels ceux de l'imam Kamagaté sur son Facebook (Binaté). L'utilisation de blagues et de légèreté dans la sphère religieuse fait écho à l'une des principales questions abordées par Meyer et Moors (2005). Elles soulignent que, bien souvent, les leaders religieux craignent les risques sociaux et moraux qu'engendrent chansons, films ou émissions de télévision et de radio. Il s'agit pour eux d'un « cheval de Troie » dans les médias religieux, incarnant l'idée que le divertissement dans le religieux pourrait détourner le croyant du message sacré et mener à une expérience religieuse plus superficielle. Cette interaction entre religion et amusement est d'autant plus frappante lorsque l'on s'informe au sujet du « Fan Club Prophète Muhammad » (Binaté; voir aussi plus haut). Qu'implique le transfert du langage de la culture commerciale populaire vers le domaine religieux? Quelles sont les formes de pratiques issues du "fandom » empruntées et reprises dans la pratique religieuse et l'adoration ? Ces questionnements connaissent un début de réponse grâce notamment à des anthropologues qui travaillent avec des réalisateurs de films commerciaux (Meyer, 2015) et des chœurs en tournée dans le monde ou au moins leur musique (Brennan, 2010).

Finalement, (c) les articles évoquent aussi la question de la religion en lien avec le visuel. Dès qu'elle entre dans la sphère publique, la religion revêt un rôle public au même titre que ses pratiquants. Ces six contributions nous invitent à penser un islam 2.0 et une chrétienté 2.0 ainsi que d'autres formes d'entités, pratiques et collectivités religieuses basées sur la participation et le partage. Dès lors que des contenus, informations et activités de nature religieuse peuvent être véhiculés ou exprimés par des personnes qui n'ont pas nécessairement une formation religieuse, de nouveaux leaders religieux peuvent émerger, et l'on peut s'interroger quant aux implications sur la pratique publique de la religion, mais aussi sur ce que signifie être une personne religieuse. À ce sujet, notons les fascinantes observations de Batibonak et Batibonak sur l'émergence de nouveaux entrepreneurs dans les modes numériques de consommation de médias religieux (aussi Graetz, 2011) ; Sylla démontre également comment les exigences des fidèles évoluent, privilégiant des pasteurs plus "high-tech» sur les 
autres. Une compétition se fait jour, et l'on attend des leaders religieux qu'ils soient actifs, mais aussi qu'ils se démarquent sur la toile.

Meyer (2006) a évoqué que, pour la chrétienté charismatique-pentecôtiste (religion de l'exposition et de la révélation), les médias constituent des espaces particulièrement favorables. En mettant un fort accent sur la lumière, l'exposition et la révélation, les auteurs de médias chrétiens font un usage habile des technologies et de l'animation (Meyer, 2015). Au contraire, quelles sont les implications pour les pratiques islamiques où l'audible est prédominant? L'analyse de Hirschkind (2006) de cassettes audio de sermons du Caire montre que l'ouïe est le sens premier par lequel l'enseignement religieux est transmis. Quel est le lien des vidéos de da'wa publiées sur YouTube ou d'autres plateformes numériques avec les politiques islamiques du corps ? Y a-t-il actuellement une reconfiguration des épistémologies de l'islam et de ses modes d'apprentissage ? Ou les leaders islamiques sont-ils en train de céder à la primauté du visuel au sein des médias sociaux ? Miller et Sinanan (2017) ont récemment avancé que la communication sur les réseaux sociaux se produit avant tout sous des formes visuelles (mèmes, photographies, émoticônes, vidéos...). L'une des questions passionnantes qui émerge pour les recherches futures est celle des langages visuels religieux numériques et leur lien avec des modes de transmission de savoirs établis, mais aussi plus récents.

\section{Une comparaison avec le religieux et le numérique à Kinshasa}

Dès 2003, j’ai mené des recherches ethnographiques dans le monde médiatique de Kinshasa, en particulier sur la production de fictions religieuses télévisées et sa connexion avec les pratiques religieuses. J'ai pu constater l'apparition des médias numériques et leur importance croissante dans les mondes vécus (lifeworlds) des Kinois, dans les paroisses au cours de sermons et dans la quête de devenir de meilleurs chrétiens. Trois autres problématiques traversent mon œuvre et apparaissent aussi dans ces six articles : l'haptique, l'interactivité comme mode religieux et l'interaction entre religion, technologie et science.

Premièrement, Batibonak et Batibonak décrivent comment les utilisateurs en ligne (par YouTube ou autres) sont appelés à toucher de l'eau tout en suivant un flux vidéo. Cette pratique existe aussi avec d'autres médias, en particulier dans des émissions radio ou télévisés. Dans Pype (2012), j’ai proposé une description ethnographique d'un show de guérison semblable, diffusé sur l'une des chaînes de télévision religieuses de Kinshasa. Il me semble que ce matériau révèle comment les leaders religieux, ainsi que les fidèles, confèrent un caractère charismatique aux médias de masse : les spectateurs contribuent activement, par des invocations, en appliquant de l'eau sur le poste de télévision, ou en tendant les mains pour toucher l'écran, dans un but de guérison, de succès ou d'enrichissement. Nous pouvons en déduire que le religieux est 
avant tout haptique, c'est dans et à travers le corps que le pouvoir et la connaissance sont transférés. Dans un tel schéma de pensée, la technologie et la religion sont incorporées dans un monde de forces mystérieuses. Par ailleurs, des forces démoniaques peuvent aussi être transférées, c'est pourquoi le leader religieux doit développer une pédagogie médiatique élaborée afin d'aider ses fidèles à vivre dans un monde saturé de médias. En effet, les leaders religieux attribuent du sens spirituel à la consommation des médias, unissant ainsi les utilisateurs avec des esprits, au sein d'une zone de mutualité (Pinney, 2002; Pype, 2012). Il y a un besoin urgent de recherches comparatives sur les moyens de constitution de ces zones de mutualité, et ces six articles ici contribuent à cet agenda. Des comparaisons sont non seulement nécessaires entre la chrétienté et l'Islam, mais également au sein même de la chrétienté (pour une comparaison entre les chrétientés kimbanguiste et pentecôtiste à Kinshasa, voir Pype, 2014) et lorsque ces dernières se rencontrent (voir par exemple les médias interreligieux au Burkina Faso). Comment cette mutualité affecte-t-elle les régimes sensoriels ? Et comment ceux-ci s'inscrivent-ils dans le tissu plus large, sensuel, d'une ville ou d'une communauté?

Deuxièmement, l'interactivité est une modalité importante de la pratique religieuse. Il a été avancé que les médias de masse charismatiques-pentecôtistes d'Afrique insistent sur la participation de l'assistance comme le fruit d'une stratégie pour garder une emprise sur la vie quotidienne des fidèles (Graetz, 2014). Néanmoins, l'idéologie (néo)pentecôtiste, qui souligne l'urgence de la bataille spirituelle, requiert une immédiateté entre pratiquants de lieux proches et plus éloignés (Pype, 2014), car tout le monde, loin ou proche, est impliqué dans le même combat spirituel.

Je suis fascinée par l'interactivité qui existe entre diverses communautés islamiques de la diaspora, et suis curieuse d'appréhender les principes religieux de l'enseignement islamique qui corroboreraient la possibilité de communications instantanées. Au-delà de ces défis, en porte-à-faux avec les formes établies de transmission de savoirs, l'interactivité sur les réseaux sociaux constitue également une source importante de débats académiques.

Finalement, le fait que des communautés d'étudiants ont pu jouer un rôle déterminant dans la portée du mouvement en ligne Hizbut-Tarqiyyah mouride (Sylla) et la sphère numérique catholique ouagalaise, n'est guère surprenant. Ces négociants IT, formés ou non en web-design ou en communication numérique, sont aussi des leaders contemporains. Dans mes propres recherches sur l'« intelligence urbaine» (Pype 2017b) à Kinshasa, j'ai observé comment les catégories de leaders mystiques et de spécialistes des technologies de l'information s'entremêlent. Je suis curieuse d'en apprendre davantage sur le positionnement socioculturel de ceux qui ont mis en ligne les pages web pour des communautés religieuses. Comment ces compétences informatiques sont-elles évaluées au sein d'un univers religieux ? Comment les pratiquants marient-ils l'intangibilité d'Internet avec le spirituel ? À Kinshasa, à tout le moins, règnent une grande confusion et une suspicion à l'égard des ingénieurs et informati- 
ciens doués. Certains pasteurs branhamistes de Kinshasa attribuent un caractère démoniaque à toute maîtrise de la technologie, la réduisant à des connaissances émanant du diable (Pype, 2017a). Leur connaissance de l'invisible est connectée à leur foi et non à des expériences scientifiques ou de déductions. Nous touchons ici le cœur de la cohabitation entre la technologie et la religion dans la modernité contemporaine. Et la question fondamentale demeure : quel type de connaissance est jugé moral ?

\section{Bibliographie}

ANDERSON B. (1983), Imagined Communities: Reflections on the Origin and Spread of Nationalism, London, Verso.

Appadurai A. (1996), Modernity at Large. Cultural Dimensions of Globalization, Minneapolis, University of Minnesota Press.

Bernal V. (2014), Nation as Network: Diaspora, Cyberspace and Citizenship, Chicago, University of Chicago Press.

BRENNAN V. (2010), « Mediating “The Voice of the Spirit”: Musical and Religious Transformations in Nigeria's Oil Boom », American Ethnologist, vol. 37, nº 2, p. 354-370.

DE WiTTE M. (2011), « Touched by the Spirit: Converting the Senses in a Ghanaian Charismatic Church », Ethnos, vol. 76, n 4, p. 489-509.

GRAETZ T. (2011), « Contemporary African mediascapes: New actors, genres and communication spaces”, Journal of African Media Studies, vol. 3, n² 2, p. 151-160.

GRAETZ T. (2014), « Christian religious radio production in Benin: The Case of Radio Maranatha », Social Compass, vol. 61, n¹0, p. 57-66.

HACKetT R. I. J., SOARES B. F. (dir.) (2014), New Media and Religious Transformations in Africa, Bloomington, Indiana University Press.

HiRSCHKIND C. (2006), The Ethical Soundscape: Casette Sermons and Islamic Counterpublics, New York, Columbia University Press.

Meyer B. (2006), " Religious Revelation, Secrecy and the Limits of Visual Representation », Anthropological Theory, vol. 6, $\mathrm{n}^{\circ}$ 4, p. 431-453.

Meyer B. (2015), Sensational Movies. Video, Vision, and Christianity in Ghana, Chicago, University of Chicago Press

Meyer B., Moors A. (dir.) (2005), Religion, Media, and the Public Sphere, Bloomington, Indiana University Press. 
Miller D., Costa E., Haynes N., McDonald T., Nicolescu R., Sinanan J., Spyer J., VenkaTRAMAN S., WANG X. (2016), How the World Changed Social Media, London, UCL Press.

MilLER D., SinANAN J. (2017), Visualising Facbook, London, UCL Press.

PINNEy C. (2002), « The Indian Work of Art in the Age of Mechanical Reproduction: Or, What Happens When Peasants “Get Hold” of Images », in F. D. GINSBURG, L. ABULughod, B. LARKIN (dir.), Media Worlds: Anthropology on New Terrain, Berkeley, University of California Press, p. 355-369.

PyPE K. (2012), The Making of the Pentecostal Melodrama. Religion, Media, and Gender, in Kinshasa, Oxford/New York, Berghahn Books.

PYPE K. (2014), « The Liveliness of Pentecostal-Charismatic Popular Culture in Africa », in M. LindHARDT (dir.), Pentecostalism in Africa. Presence and Impact of Pneumatic Christianity in Postcolonial Societies, Leiden, Brill, p. 345-378.

PyPe K. (2017a), « Branhamist Kindoki: Ethnographic Notes on Connectivity, Technology and Urban Witchcraft in Contemporary Kinshasa », in K. RIO, M. MACCARTHY, R. Blanes (dir.), Pentecostalism and Witchcraft in Melanesia and Africa, London, Palgrave MacMillan, p. 110-144.

PyPE, K. (2017b) « Smartness from Below. Variations on Technology and Creativity in Contemporary Kinshasa », in C.C. MAVHungA (dir.), What do Science, Technology, and Innovation Mean from Africa?, MIT Press, p. 97-115.

PyPE K. (2018), « Nzete Ekauka versus the Catholic Church. Religious Competition, Media Ban and the Virgin Mary in Contemporary Kinshasa », in F. BECKER, J. CABrita, M. Rodet (dir.), Religion, Media, Marginality in Modern Africa, Ohio University Press, p. 202-228.

Schulz D. (2011), Muslims and New Media in West Africa. Pathways to God, Bloomington, Indiana University Press. 